ORIGINAL ARTICLE

\title{
Effects of dietary carbohydrate on delayed onset muscle soreness and reactive oxygen species after contraction induced muscle damage
}

\author{
G L Close, T Ashton, T Cable, D Doran, C Noyes, F McArdle, D P M MacLaren
}

Br J Sports Med 2005;39:948-953. doi: 10.1136/bjsm.2005.019844

See end of article for authors' affiliations

\section{Correspondence to:} Dr Close, Clinical Sciences, University of Liverpool, Liverpool L69 3GA, UK; gclose@liv.ac.uk

Accepted 17 May 2005

\begin{abstract}
Background: Delayed onset muscle soreness (DOMS) occurs affer unaccustomed exercise and has been suggested to be attributable to reactive oxygen species (ROS). Previous studies have shown increased ROS after lengthening contractions, attributable to invading phagocytes. Plasma glucose is a vital fuel for phagocytes, therefore carbohydrate $(\mathrm{CHO})$ status before exercise may influence ROS production and DOMS

Objective: To examine the effect of pre-exercise $\mathrm{CHO}$ status on DOMS, ROS production, and muscle function after contraction induced muscle damage.

Method: Twelve subjects performed two downhill runs, one after a high $\mathrm{CHO}$ diet and one after a low $\mathrm{CHO}$ diet. Blood samples were drawn for analysis of malondialdehyde, total glutathione, creatine kinase, non-esterified fatty acids, lactate, glucose, and leucocytes. DOMS and muscle function were assessed daily.

Results: The high $\mathrm{CHO}$ diet resulted in higher respiratory exchange ratio and lactate concentrations than the low $\mathrm{CHO}$ diet before exercise. The low $\mathrm{CHO}$ diet resulted in higher non-esterified fatty acid concentrations before exercise. DOMS developed after exercise and remained for up to 96 hours, after both diets. A biphasic response in creatine kinase occurred after both diets at 24 and 96 hours after exercise. Malondialdehyde had increased 72 hours after exercise after both diets, and muscle function was attenuated up to this time.

Conclusions: Downhill running resulted in increased ROS production and ratings of DOMS and secondary increases in muscle damage. $\mathrm{CHO}$ status before exercise had no effect.
\end{abstract}

during exercise, resulting in a greater stress response and an associated immunosuppression.

The contribution of phagocytes to ROS production after exercise and the role of ROS in the aetiology of DOMS and loss of muscle function are unclear. Likewise, despite the popular practice by athletes of altering their $\mathrm{CHO}$ status before exercise to maximise performance, the effect of such dietary manipulations on DOMS, muscle function, and ROS production has not been reported. Therefore the main aims of this study were to: (1) investigate the effects of alterations in CHO status before exercise on phagocyte derived ROS production; (2) clarify the effects of dietary CHO manipulation on DOMS and muscle function.

\section{METHODS \\ Subjects}

Twelve physically active male subjects of mean (SEM) age 23.3 (0.98) years, height $175(1.56) \mathrm{cm}$, body mass 76.7 (1.73) kg, maximal oxygen uptake ( $\left.\mathrm{VO}_{2} \mathrm{MAx}\right) 4.2$ (0.14) litres/ min, and body fat 14.6 (1.06)\%, who were naïve to downhill running, volunteered for the study. All were informed verbally and in writing about the nature of the study, including all potential risks. Written consent was obtained before participation, and ethical approval was granted by the ethics committee of Liverpool John Moores University.

Abbreviations: $\mathrm{CHO}$, carbohydrate; $\mathrm{CK}$, creatine kinase; DOMS, delayed onset muscle soreness; MDA, malondialdehyde; NEFA, nonesterified fatty acids; RER, respiratory exchange ratio; ROS, reactive oxygen species; $\mathrm{VO}_{2} \mathrm{MAX}$, maximal oxygen uptake 
Table 1 Characteristics of the high carbohydrate $(\mathrm{HC})$ and low carbohydrate (LC) diets

\begin{tabular}{lll}
\hline & HC & LC \\
\hline Fat (kJ) & 1536 & 11126 \\
CHO (kJ) & 11214 & 1619 \\
Protein (kJ) & 1760 & 1783 \\
Total energy (kJ) & 14510 & 14528 \\
Fat (g) & 40 & 282 \\
Carbohydrate (g) & 620 & 80 \\
Protein (g) & 75 & 75 \\
\hline & & \\
\hline
\end{tabular}

\section{Experimental protocol}

The aerobic fitness of each subject was initially assessed by determining their $\mathrm{VO}_{2} \mathrm{MAX}$ as previously. ${ }^{3}$ This assessment was carried out one week before the first experimental run. All subjects then performed two downhill runs, both lasting 30 minutes at a running speed corresponding to $60 \% \mathrm{VO}_{2} \mathrm{MAX}$. One run was performed after two days on a high carbohydrate diet (HC), and the other after two days on a low carbohydrate diet (LC). All subjects were randomly allocated to one of two groups: LC first or HC first. The conditions were counterbalanced, and there was a five week interval between trials.

For each trial subjects were required to visit the laboratory on five consecutive days. On day 1 they performed the experimental run, had leg torque and pain measurements taken, and gave venous blood samples before and after the run. On the following four days, they had torque and pain assessed and gave a resting venous blood sample.

\section{Dietary intervention}

All subjects followed a given diet for 48 hours before the experimental trials. The diets were $\mathrm{HC}(77 \% \mathrm{CHO}, 12 \%$ protein, $11 \%$ fat) and LC (11\% CHO, 12\% protein, $77 \%$ fat). They were designed for each subject ensuring that $\mathrm{HC}$ contained $\sim 8 \mathrm{~g} / \mathrm{kg}$ body weight $\mathrm{CHO}$ and LC contained $\sim 1 \mathrm{~g} / \mathrm{kg}$ body weight. The diets were produced in consultation with the subjects to ensure palatability. Fluids were consumed ad libitum, although drinks containing sugar were excluded. The diets were iso-energetic, with both diets providing $\sim 14 \mathrm{MJ}$ of energy. Table $\mathrm{l}$ summarises the characteristics of the two diets.

\section{Day 1}

Subjects arrived at the laboratory after an overnight fast, and a blood sample was taken before the run. All blood samples were collected into vacutainers with a total of three vacutainers at each sample point. Tube 1 contained EDTA and was used for the analysis of leucocytes, total glutathione, and lactate. Tube 2 contained serum separation gel and was used for the analysis of malondialdehyde (MDA). Tube 3 contained lithium heparin and was used for the analysis of non-esterified fatty acids (NEFA), glucose, and creatine kinase (CK).

Glucose, CK, and NEFA were measured by enzymatic methods using a commercially available kit (Instrumentation Laboratory, Warrington, Lancashire, UK) on an IL Lab 300 chemistry analyser. Lactate was immediately deproteinised using cold perchloric acid and later analysed on an IL Lab 300 chemistry analyser using a commercially available kit (Randox Lactate PAP; Randox Laboratories, Crumlin, Co Antrim, UK). MDA was analysed by high performance liquid chromatography (Gino 50; Dionex, Macclesfield, Cheshire, UK) using the methods of Esterbaur et al. ${ }^{11}$ Total glutathione was measured spectrophotometrically using a 96 well microplate reader with kinetics by the methods of Anderson. ${ }^{12}$ Total and differential leucocytes, haemoglobin, and packed cell volume were determined on an automated Coulter counter (Coulter MAXM analyser; Coulter Corporation, Miami, Florida, USA).

After the blood sample had been taken, subjects were asked to rate the pre-exercise muscle soreness of their gastrocnemius, tibialis anterior, hamstring, quadriceps, gluteal (both left and right sides), and lower back muscles using a visual analogue scale. Briefly, they were asked to contract the selected muscle and then make a mark along a $10 \mathrm{~cm}$ line. The far left point of the line represented no pain at all and the far right point represented unbearable pain. The distance from no pain at all was measured, and this represented the magnitude of DOMS for that particular muscle. Scores were recorded as the mean pain for the 11 assessed sites. Muscle function was then assessed concentrically and eccentrically using an isokinetic leg dynamometer as described previously. ${ }^{3}$

The downhill run was performed on the same motorised treadmill as used in the $\mathrm{VO}_{2} \mathrm{MAX}$ test. The run lasted for 30 minutes at an oxygen consumption corresponding to $60 \%$ $\mathrm{VO}_{2}$ MAX. Respiratory exchange ratio (RER) and $\mathrm{VO}_{2}$ were assessed every 10 seconds throughout the run using online

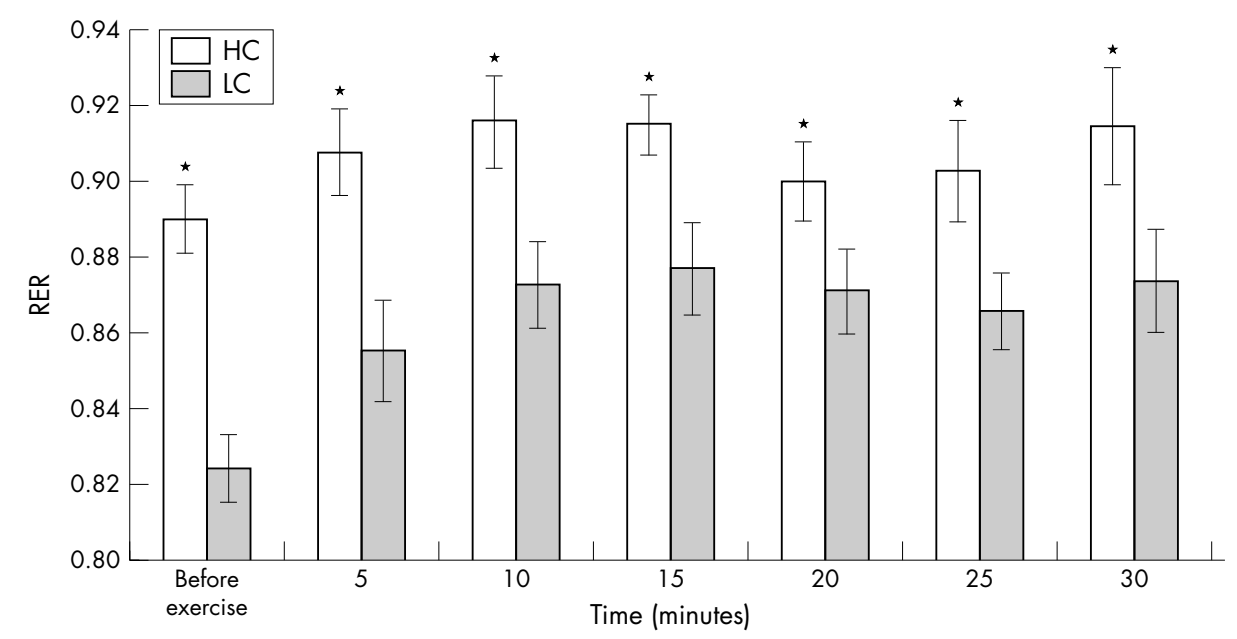

Figure 1 Mean (SEM) non-protein respiratory exchange ratio (RER) before and during a downhill treadmill run after two days on a high carbohydrate diet $(H C)$ or two days on a low carbohydrate diet $(L C)$. ${ }^{*}$ Significant difference from LC $(p<0.05)$. 
Table 2 Metabolite data obtained before and after a downhill run performed after consumption for two days of a high carbohydrate $\operatorname{diet}(\mathrm{HC})$ or a low carbohydrate diet (LC)

\begin{tabular}{|c|c|c|c|c|}
\hline \multirow[b]{2}{*}{ Metabolite } & \multicolumn{2}{|c|}{ Before exercise } & \multicolumn{2}{|l|}{ After exercise } \\
\hline & $\mathrm{HC}$ & LC & $\mathrm{HC}$ & LC \\
\hline Glucose (mM) & $5.2(0.18)$ & $4.6(0.17)$ & $6.3(0.29)^{*} \dagger$ & $4.9(0.23)$ \\
\hline Lactate (mM) & $1.9(0.18) \dagger$ & $1.3(0.09)$ & $2.7(0.25)^{*}$ & $2.3(0.27)^{\star}$ \\
\hline NEFA (mM) & $0.17(0.02) \dagger$ & $0.38(0.08)$ & $0.52(0.07) \dagger$ & $0.81(0.11)^{*}$ \\
\hline \multicolumn{5}{|c|}{$\begin{array}{l}\text { Values are mean (SEM). } \\
\text { *Significant difference from before exercise }(p<0.05) \text {. } \\
\text { †Significant difference from } L C(p<0.05) \text {. } \\
\text { NEFA, Non-esterified fatty acids. }\end{array}$} \\
\hline
\end{tabular}

gas analysis (Metamax Cortex Biophysic GMbH, Leipzig, Germany).

Immediately after the run, subjects lay down and a second venous blood sample was taken. Their perceptions of muscle soreness were reassessed before they completed the force assessment again.

\section{Days 2, 3, 4, and 5}

Subjects visited the laboratory exactly $24,48,72$, and 96 hours after their initial visit. They were seated for 30 minutes before a resting venous blood sample was taken from a prominent vein. The blood test took place at the same time of day as the pre-exercise blood test performed on the initial day to eliminate any circadian effects. ${ }^{13}$ After the blood tests, subjects were assessed for DOMS. This was followed by a five minute warm up on a cycle ergometer before muscle torque was reassessed. All subjects were then allowed five weeks of recovery before they repeated the test using the second dietary condition.

\section{Statistical analysis}

Statistical analysis was carried out using the Statistical Package for Social Sciences (SPSS Surrey, UK). All data are presented as mean (SEM). Data were analysed using a repeated measures analysis of variance to prevent familywise error. When Mauchley's test of sphericity indicated a minimal level of violation $(>0.75)$, the degrees of freedom was corrected using the Huynh-Feldt adjustment. When the sphericity was $<0.75$, the Greenhouse-Geisser correction was used. $^{14}$ Post hoc Tukey analysis (Honestly Significant Difference (HSD)) was performed to identify where the significant differences occurred. Significance was set at the $\alpha$ level of 0.05 for all tests.

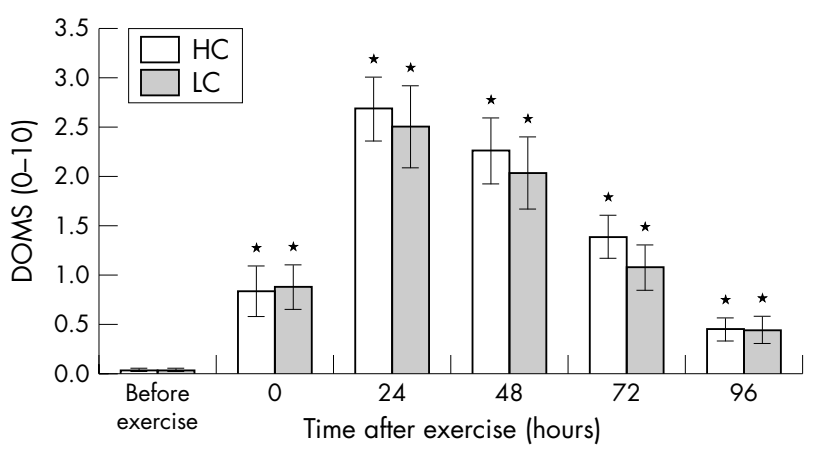

Figure 2 Mean (SEM) ratings of delayed onset muscle soreness (DOMS), measured using a visual analogue scale, before and after a downhill treadmill run performed after two days on a high carbohydrate $\operatorname{diet}(\mathrm{HC})$ or two days on a low carbohydrate $\operatorname{diet}(\mathrm{LC})$. *Significant difference from before exercise $(p<0.05)$.

\section{RESULTS}

All subjects successfully completed the 30 minute run on both occasions, and were able to maintain $60 \% \mathrm{VO}_{2} \mathrm{MAX}$ throughout. There was no significant difference in mean $\mathrm{VO}_{2}$, rating of perceived exertion, heart rate, minute ventilation, and run speed between the two dietary interventions $(\mathrm{p}>0.05$, data not presented).

\section{Metabolite data}

Figure 1 shows non-protein RER during the runs under both dietary conditions. HC resulted in a significantly higher RER before exercise, and this remained elevated throughout the duration of the run. Mean RERs throughout the runs were $0.91(0.01)$ and 0.85 (0.01) for HC and LC respectively.

Plasma glucose, NEFA, and lactate concentrations were measured immediately before and after exercise (table 2 ). Before exercise, there was no significant difference in blood glucose between the dietary interventions. However, HC produced a significant $(\mathrm{p}<0.05)$ increase in plasma glucose
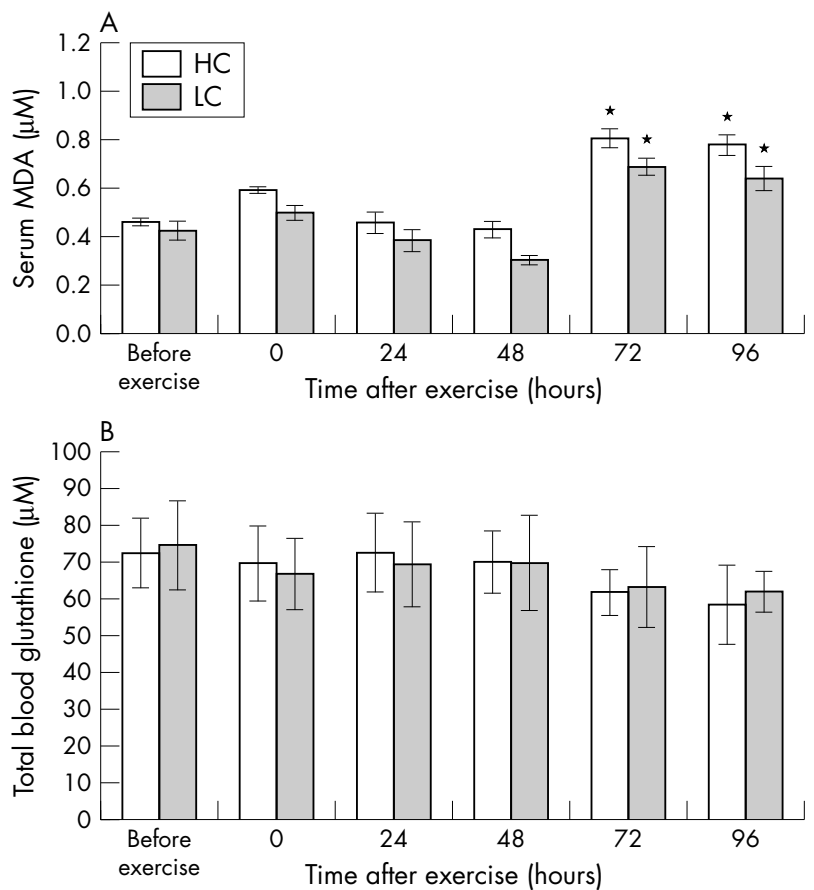

Figure 3 (A) Mean (SEM) serum malondialdehyde (MDA) concentrations and (B) mean (SEM) total blood glutathione concentrations before and after a downhill treadmill run performed after two days on a high carbohydrate diet $(\mathrm{HC})$ or two days on a low carbohydrate diet (LC). *Significant difference from before exercise $(p<0.05)$. 


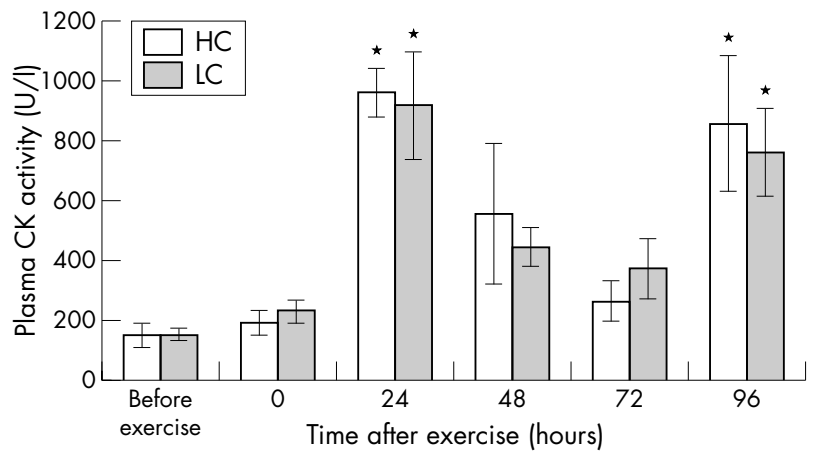

Figure 4 Mean (SEM) plasma creatine kinase (CK) activity before and after a downhill treadmill run performed after two days on a high carbohydrate diet $(\mathrm{HC})$ or two days on a low carbohydrate diet (LC). *Significant difference from before exercise $(p<0.05)$.

immediately after exercise whereas LC failed to do so $(p>0.05)$. Before exercise, NEFA concentrations were significantly $(\mathrm{p}<0.05)$ higher after LC than after HC. Furthermore, there was a significant $(p<0.05)$ increase in NEFA concentration after exercise for both dietary conditions, although the rise after LC was significantly $(p<0.05)$ greater than after HC.

Before exercise, lactate concentrations were significantly $(\mathrm{p}<0.05)$ higher after $\mathrm{HC}$ than after LC. Both dietary conditions resulted in a significant $(p<0.05)$ increase in lactate after exercise, although there was no significant difference between the conditions.

\section{Delayed onset muscle soreness}

For both dietary conditions, there was a significant $(\mathrm{p}<0.05)$ increase in ratings of muscle soreness immediately after exercise that cannot be classed as DOMS. However, there was a further significant increase 24 hours after exercise, and this remained elevated for up to 96 hours $(\mathrm{p}<0.05)$ for both conditions. There were no significant differences in DOMS between the two conditions (fig 2).

\section{Reactive oxygen species}

Serum MDA and total blood glutathione were used as indicators of ROS activity (fig 3 ). There were no significant increases in MDA after exercise $(p<0.05)$ although it had increased significantly $(p<0.05)$ in both groups 72 hours after exercise and remained raised at 96 hours. There were no significant differences between the two dietary interventions. There was no significant main effect of time or group on total blood glutathione concentrations (fig 3B).

\section{Muscle damage}

Plasma CK activity was used to assess muscle damage (fig 4). There was a significant $(\mathrm{p}<0.05)$ increase in plasma CK activity 24 hours after exercise for both dietary conditions. This had fallen by 48 hours and returned to baseline levels at 72 hours after exercise. There was, however, a second significant $(\mathrm{p}<0.05)$ peak in CK 96 hours after exercise, although there was no significant difference between the two conditions.

\section{Muscle function}

Figure 5A shows the change in concentric torque at $1.04 \mathrm{rad} / \mathrm{s}$ after both dietary interventions. There was a significant reduction in muscle torque 24 hours after exercise, and this remained suppressed 72 hours after exercise, although there was no significant differences between the dietary conditions.
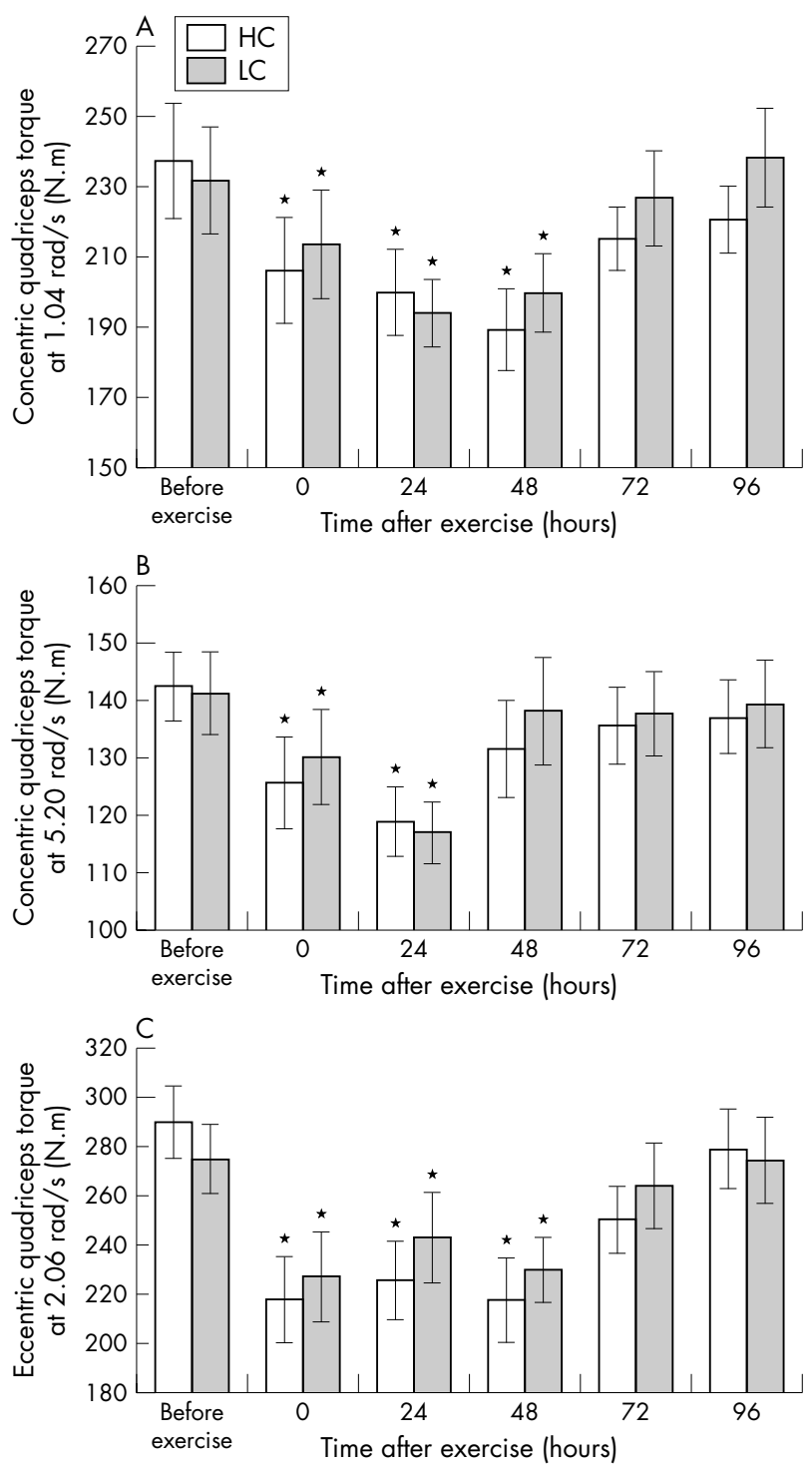

Figure 5 Mean (SEM) concentric quadriceps torque measured at (A) $1.04 \mathrm{rad} / \mathrm{s}$, (B) $5.20 \mathrm{rad} / \mathrm{s}$, or (C) $2.06 \mathrm{rad} / \mathrm{s}$ before and after a downhill treadmill run performed after two days on a high carbohydrate $\operatorname{diet}(\mathrm{HC})$ or two days on a low carbohydrate diet (LC). *Significant difference from before exercise $(p<0.05)$.

Concentric quadriceps torque was also assessed at $5.20 \mathrm{rad} /$ $\mathrm{s}$ (fig 5B). There were significant $(\mathrm{p}<0.05)$ torque losses immediately and 24 hours after exercise, although muscle torque had returned to baseline by 48 hours.

Muscle torque was also assessed using an eccentric protocol (fig $5 \mathrm{C})$. There was a significant $(\mathrm{p}<0.05)$ reduction in muscle torque immediately after exercise and up to 72 hours, although there was no significant difference between the dietary conditions.

\section{Leucocytes}

Downhill running resulted in a transient leucocytosis $(\mathrm{p}<0.05)$ occurring immediately after exercise for both diets. This was largely due to transient neutrophilia $(\mathrm{p}<0.05)$ as well as a transient increase in lymphocytes $(p<0.05)$ (fig 6). There was no change in circulating eosinophil or basophil counts after either dietary condition. Furthermore, there was no significant difference in total or differential leucocyte counts between the two diets. 

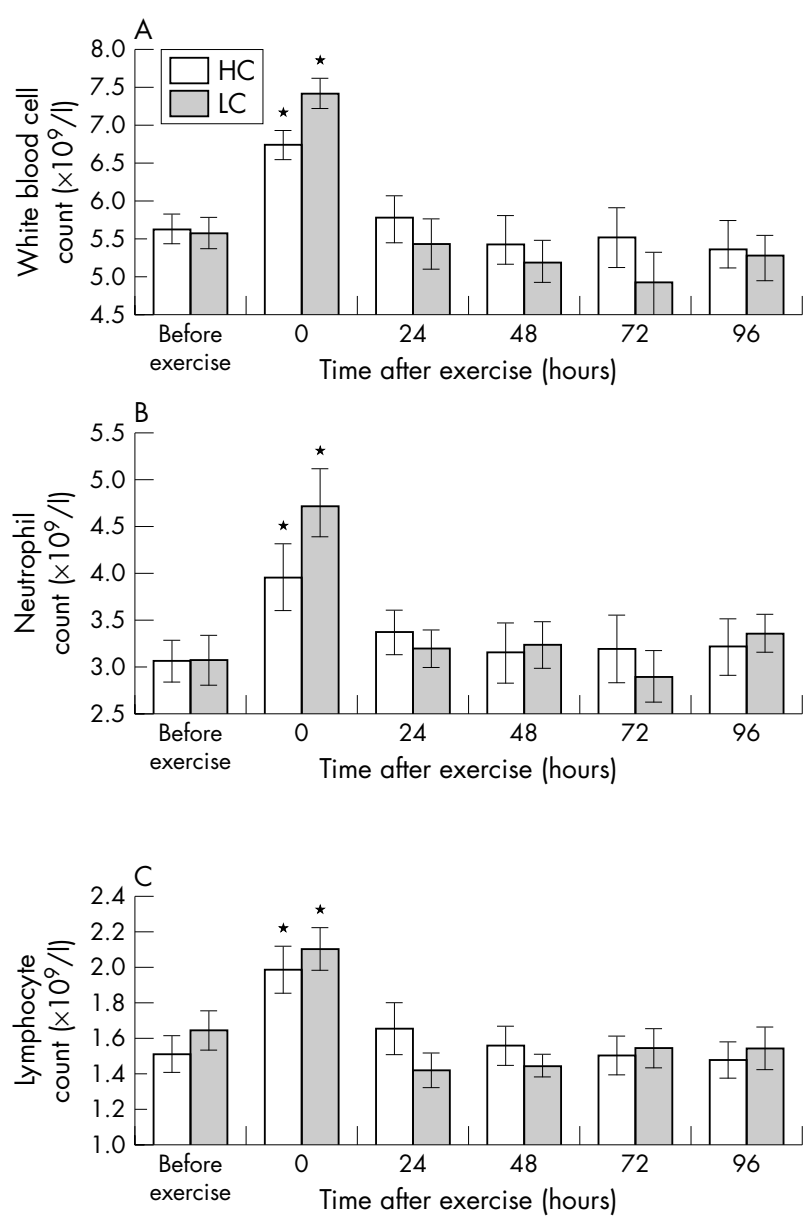

Figure 6 (A) Mean (SEM) total circulating leucocyte count, (B) mean (SEM) circulating neutrophil count, and (C) mean (SEM) circulating lymphocyte count before and after a downhill treadmill run performed after two days on a high carbohydrate diet $(\mathrm{HC})$ or two days on a low carbohydrate diet (LC). *Significant difference from before exercise $(p<0.05)$

\section{DISCUSSION}

This study investigated the effects of pre-exercise dietary CHO status on DOMS, ROS, and muscle function after contraction induced muscle damage. The downhill running protocol successfully resulted in increased ratings of DOMS and changes in muscle function and caused a delayed increase in ROS production. However, the dietary manipulation had no significant effects on DOMS, ROS, or muscle function.

Muscle damage was assessed from plasma CK activity, with no discernible differences between the two dietary conditions. This would suggest that alteration of glycogen status before exercise has no effect on muscle damage after exercise. It was interesting to observe that there was a biphasic response in CK after both dietary conditions. There was an initial increase in CK activity 24 hours after exercise, probably reflecting muscle damage caused by the mechanical trauma of the lengthening contractions. ${ }^{15}$ However, consistent with previous research, ${ }^{16}$ there was also a secondary increase in CK at 96 hours. As there was an increase in ROS production at 72 and 96 hours, it is possible that this secondary increase in CK represents ROS attack on lipid membranes resulting in secondary muscle damage. Previous studies in mice have also suggested that secondary muscle damage after lengthening muscle contractions is caused by ROS. ${ }^{17} 18$ Interestingly, the study by Zerba et al ${ }^{18}$ clearly showed that treatment with "polyethylene-glycol superoxide dismutase" prevented the secondary losses of muscle force, adding further support to the theory that secondary muscle damage may be ROS mediated.

There was, however, no relation between this secondary increase in CK activity and DOMS. DOMS had fallen significantly by 96 hours after exercise and had almost returned to pre-exercise levels. This suggests that the secondary muscle damage, possibly caused by upregulation of ROS, does not prolong the sensation of DOMS. One of the aims of this study was to determine if the production of ROS played a pathological or physiological role in the aetiology of DOMS. As the increase in ROS was not associated with further losses of muscle function or an increase in DOMS, a dissociation between ROS and DOMS is suggested. ROS were peaking at a time when muscle function and ratings of DOMS were returning to pre-exercise values, suggesting that ROS produced by phagocytic cells in the days after lengthening contractions may play a physiological role in mediating the recovery. There was also no significant difference in ratings of DOMS between the two dietary conditions, suggesting that $\mathrm{CHO}$ status before exercise has no affect on muscle soreness.

This study also sought to determine the effects of dietary CHO manipulation on muscle function after muscle damaging exercise. There were significant losses of muscle function after both diets. However, there were no significant differences between the two dietary conditions, suggesting that pre-exercise $\mathrm{CHO}$ status has no effect on muscle function after contraction induced muscle damage.

It has been suggested that high fat diets, especially those high in $\mathrm{n}-3$ polyunsaturated fatty acids, may inhibit neutrophil chemotaxis and the subsequent generation of superoxide by upregulation of superoxide dismutase. ${ }^{19}$ Although the amount of polyunsaturated fatty acids was not determined in this study, it can be concluded that two days on a $77 \%$ fat diet does not significantly inhibit the production of superoxide. Furthermore, we can also conclude from this study that high fat diets offer no protection against DOMS, as suggested by Lenn et al, ${ }^{20}$ or affect the recovery of muscle function.

Circulating leucocytes showed a similar response to that reported by Bishop et $a^{21}$ after three days on a CHO manipulated diet. There was a trend (albeit not significant) for attenuation of leucocytosis after exercise performed after the high $\mathrm{CHO}$ diet compared with the low CHO diet, which was largely due to attenuated neutrophilia. Interestingly, despite this attenuation of neutrophilia, there was no significant difference in ROS responses between the two conditions. The serum MDA and total blood glutathione concentrations suggested that oxidative stress and lipid peroxidation occurred 72 and 96 hours after exercise. One interpretation of these findings is that ROS produced in the days after downhill running are not related to post-exercise neutrophilia; however, as reported by Bishop et al, ${ }^{21}$ this type of conclusion can only be made if the functional capacity of the blood compartment is assumed to be directly related to the tissue in question, in this case the damaged muscle. One concern of this conclusion is that, in response to physical trauma, neutrophils migrate to the site of injury where they release chemotactic factors, which subsequently recruit other neutrophils to the damaged tissue. ${ }^{22}$ Moreover, it is known that, after lengthening muscle contractions, there is compartmentalisation of muscle damage, and therefore the site of injury will have a finite size. ${ }^{23}$ Therefore not all of the neutrophils in the circulation will need to infiltrate the damaged tissue and hence the circulating neutrophil count may not be a good indicator of neutrophil functional activity. 


\section{What is already know on this topic}

- DOMS occurs after lengthening contractions, and one of the proposed mechanisms for this involves ROS, presumably derived from invading phagocytes

- Phagocytes depend on plasma glucose, and therefore alteration of glycogen content before exercise may affect DOMS

\section{What this study adds}

- This study shows that two days on a low carbohydrate diet before exercising has no effect on DOMS, loss or recovery of muscle function, or production of ROS despite the vital role that glucose plays as a metabolic fuel

Bishop et $a^{21}$ reported that despite the attenuated neutrophilia associated with a high $\mathrm{CHO}$ diet, when changes in neutrophil were expressed as lipopolysaccharide stimulated elastase release per neutrophil, there was no difference between a high $\mathrm{CHO}$ and low $\mathrm{CHO}$ diet. Therefore, in relation to the present study, it would appear that, despite the trend towards attenuation of circulating neutrophils, there were still sufficient neutrophils in the circulation to migrate to the site of injury and exhibit a respiratory burst, as shown by the increased MDA production.

The subjects chose their own foods from a comprehensive list. Food was purchased and prepacked for the subjects, and the metabolite data suggest good adherence to the diets. The diets were iso-energetic and therefore any differences observed were due to the macronutrient differences and not total energy intake. Two days on a high $\mathrm{CHO}$ diet significantly increased resting RER and lactate concentrations and decreased NEFA concentrations before exercise, suggesting that the diets were successful in altering preexercise CHO status. As the diets were not combined with a bout of strenuous exercise, it is unlikely that muscle glycogen stores were significantly affected, although there was likely to have been a significant reduction in liver glycogen stores. However, despite these presumed changes in pre-exercise liver glycogen status, there were no observed differences between the two dietary interventions with regard to DOMS, ROS, or changes in muscle function. It must be noted, however, that the study did not measure liver or muscle glycogen and therefore any changes in glycogen status are deduced from the metabolic measurements. Furthermore, the dietary manipulation was only carried out for two days, and therefore conclusions on long term dietary $\mathrm{CHO}$ manipulation cannot be made.

In conclusion, 30 minutes of downhill running at $60 \%$ $\mathrm{VO}_{2} \mathrm{MAX}$ results in a delayed increase in ROS production and muscle damage. However, despite the vital role of glucose as a metabolic fuel, the two day alteration in $\mathrm{CHO}$ status before the exercise had no effect on DOMS, muscle function, or ROS production.

\section{ACKNOWLEDGEMENTS}

We thank Dr Anne McArdle, Professor Malcolm Jackson (University of Liverpool), and Professor Michael Gleeson (Loughborough University) for useful scientific discussions, and all of the subjects for their efforts.

\section{Authors' affiliations}

G L Close, T Cable, D Doran, C Noyes, D P M MacLaren, Liverpool John Moores University, Liverpool, UK

T Ashton, F McArdle, University of Liverpool, Liverpool

Competing interests: none declared

\section{REFERENCES}

1 Gullick DT, Kimura IF. Delayed onset muscle soreness: what is it and how do we treat it? Journal of Sport Rehabilitation 1996;5:234-43.

2 Newham DJ, Mills KR, Quigley BM, et al. Pain and fatigue after concentric and eccentric muscle contractions. Clin Sci (Lond) 1983;64:55-62.

3 Close GL, Ashton T, Cable T, et al. Eccentric exercise, isokinetic muscle torque and delayed onset muscle soreness: the role of reactive oxygen species. Eur J Appl Physiol 2004:91:615-21.

4 Eston RG, Pears J, Jackson M. Association between the production of thiobarbituric reactive substances (malondialdehyde) and markers of muscle damage induced by uphill and downhill running. J Sports Sci 1996;14:80P.

5 Gleeson M, Bishop NC. Elite athlete immunolgy: importance of nutrition. Int J Sports Med 2000;21:S44-50.

6 Bishop NC, Blannin AK, Walsh NP, et al. Carbohydrate beverage ingestion and neutrophil degranulation responses following cycling to fatigue at $75 \%$ VO2 max. Int J Sports Med $2001 ; 22: 226-31$

7 Bishop NC, Blannin AK, Walsh NP, et al. Nutritional aspects of immunosupression in athletes. Sports Med 1999;28:151-76.

8 Gleeson M, Blannin AK, Walsh NP, et al. Effect of low- and highcarbohydrate diets on the plasma glutamine and circulating leukocyte responses to exercise. Int $J$ Sport Nutr 1998;8:49-59.

9 Henson DA, Nieman DC, Blodgett AD, et al. Influence of exercise mode and carbohydrate on the immune responses to prolonged exercise. Int J Sports Nutri 1999;9:213-28.

10 Nieman DC, Nehlsen-Cannarella SL, Fagoaga OR, et al. Effects of mode and carbohydrate on the granulocyte and monocyte response to intensive prolonged exercise. J Appl Physiol 1998;84:1252-9.

11 Esterbaur H, Lang J, Zadravec S, et al. Detection of malonaldehyde by high performance liquid chromatography. Methods Enzymol 84, 105:319-28.

12 Anderson M. Determination of glutathione and glutathione disulphide. Methods Enzymol 1985; 113:548-55.

13 Fernandes $\mathbf{G}$. Chronobiology of immune functions: cellular and humoral aspects. In: Touitou Y, Haus E, eds. Biologic rhythms in clinical and laboratory medicine. Berlin: Springer-Verlag, 1994:493-503.

14 Field A. Discovering statistics using SPSS for Windows. Discovering statistics using SPSS for windows. London: Sage Publications, 1999.

15 Clary S, Schwane JA. Responses of serum CK, LDH, and LDH-I activities to exercise with a large eccentric component. Med Sci Sports Exerc 1988:20:S74

16 Smith LL, Gond JA, Holbert D, et al. Differential white cell counts after two bouts of downhill running. Int J Sports Med 1998;19:432-7

17 McArdle A, Van Der Meulen JH, Catapano M, et al. Free radical activity following contraction-induced injury to the extensor digitorum longus muscles of rats. Free Radic Biol Med 1999;26:1085-91.

18 Zerba E, Komorowski TE, Faulkner JA. Free radical injury to skeletal muscles of young, adult, and old mice. Am J Physiol 1990;258:C429-35.

19 Luostarinen R, Saldeen T. Dietary fish oil decreases superoxide generation by human neutrophils: relation to cyclooxygenase pathway and lysosomal enzyme release. Postoglandins Leukot Essent Fatty Acids 1996;55:167-72.

20 Lenn J, Uhl T, Mattacola C, et al. The effects of fish oil and isoflavones on delayed onset muscle soreness. Med Sci Sports Exerc 2002;34:1605-13.

21 Bishop NC, Walsh NP, Haines DL, et al. Pre-exercise carbohydrate status and immune responses to prolonged cycling. I. Effect on neutrophil degranulation. Int J Sport Nutr Exerc Metab 2001; 1 1:490-502.

22 Gleeson M, Bishop NC. Special feature for the Olympics: effects of exercise on the immune system: modification of immune responses to exercise by carbohydrate, glutamine and anti-oxidant supplements. Immunol Cell Biol 2000;78:554-61.

23 Evans WJ. Exercise-induced skeletal muscle damage. Phys Sportsmed 1987;15:89-100. 